\title{
The beauty of the electromagnetic probe
}

\author{
R.G. Milner ${ }^{\mathrm{a}}$ \\ 02139, USA \\ Published online: 15 May 2006 - C Società Italiana di Fisica / Springer-Verlag 2006
}

MIT-Bates Linear Accelerator Center, Laboratory for Nuclear Science, Massachusetts Institute for Technology, Cambridge, MA

\begin{abstract}
Precision experiments using the electromagnetic probe have recently produced important new data on fundamental properties of the nucleon, e.g. charge, magnetism, shape, polarizability, spin and sea quark structure. These experiments have been made possible by a new generation of high duty factor electron accelerators, advances in spin polarization technology (beams, targets and recoil polarimeters), and the development of unique, optimized detector systems. In this contribution, the role of multiple photon exchange in electron scattering from the proton and the role of sea quarks in nucleon structure are highlighted.
\end{abstract}

PACS. 13.40.Gp Electromagnetic form factors - 13.60.-r Photon and charged-lepton interactions with hadrons - 13.60.Fz Elastic and Compton scattering - 14.20.Dh Protons and neutrons

\section{Introduction}

Understanding the structure of the nucleon in terms of the fundamental constituents of the Standard Model, the quarks and gluons of Quantum Chromodynamics (QCD), is a major research area in Physics. The ultimate goal is to test QCD with precision measurements and $a b$ initio calculations. Over the last decade, experimentalists have made substantial progress in determination of the quark and gluon distributions at high energies $\left(E_{C M} \sim\right.$ $100 \mathrm{GeV}$ ) and measurement of fundamental properties of the nucleon at low energies $\left(E_{C M} \sim 1 \mathrm{GeV}\right)$. Theorists are starting to produce full QCD Monte Carlo simulations (albeit with heavy pion masses) of nucleon structure using advanced computers [1].

The experimental study of the structure of the proton and of atomic nuclei is best carried out using the pointlike electroweak probe, the best understood interaction in Nature. Intense beams of highly polarized electrons have become available at energies of 0.5 to $6 \mathrm{GeV}$ at high duty factor. Highly polarized proton, deuteron and ${ }^{3} \mathrm{He}$ targets have been developed as well as efficient polarimeters for detection of recoil polarization. Optimized experiments utilizing uniquely designed detectors have been carried out. New data and insights have been obtained in measurement of the following properties of the nucleon:

- The proton and neutron charge and magnetism through spin-dependent elastic electron scattering at Mainz [2], Bates [3], NIKHEF [4] and JLab [5]. Precise measurements of all four of the nucleon elastic

\footnotetext{
a e-mail: milner@mit.edu
}

form-factors have been carried out. In particular, the relatively small neutron electric form-factor has been determined to better than $7 \%$ over the range $0.1<Q^{2}<2(\mathrm{GeV} / c)^{2}$.

- The shape of the proton through study of electroexcitation of the $\pi^{0}$ at the $\Delta(1232)$-resonance at low $Q^{2} \sim 0.1(\mathrm{GeV} / c)^{2}$ using out-of-plane detection at Bates and Mainz [6]. It has been established that the proton shape is slightly non-spherical. A chiral extrapolation [7] of lattice QCD calculations [8] is in good agreement with the data.

- The electric and magnetic polarizabilities of the proton through measurement of Virtual Compton Scattering from the proton at Mainz [9] and JLab [10] and using out-of-plane detection at Bates [11].

- The quark and gluon contributions to the spin structure of the proton using deep inelastic scattering at HERMES/DESY [12], JLab [13], COMPASS/CERN [14] and RHIC-spin [15].

- The role of strange quarks in the long distance magnetic and electric charge distribution of the proton at Bates, Mainz and JLab $[16,17]$. There are hints of a non-zero strange quark magnetic moment of the proton but these need to be confirmed by more precise experiments.

Here I concentrate on two areas of research where important results have recently been obtained. 


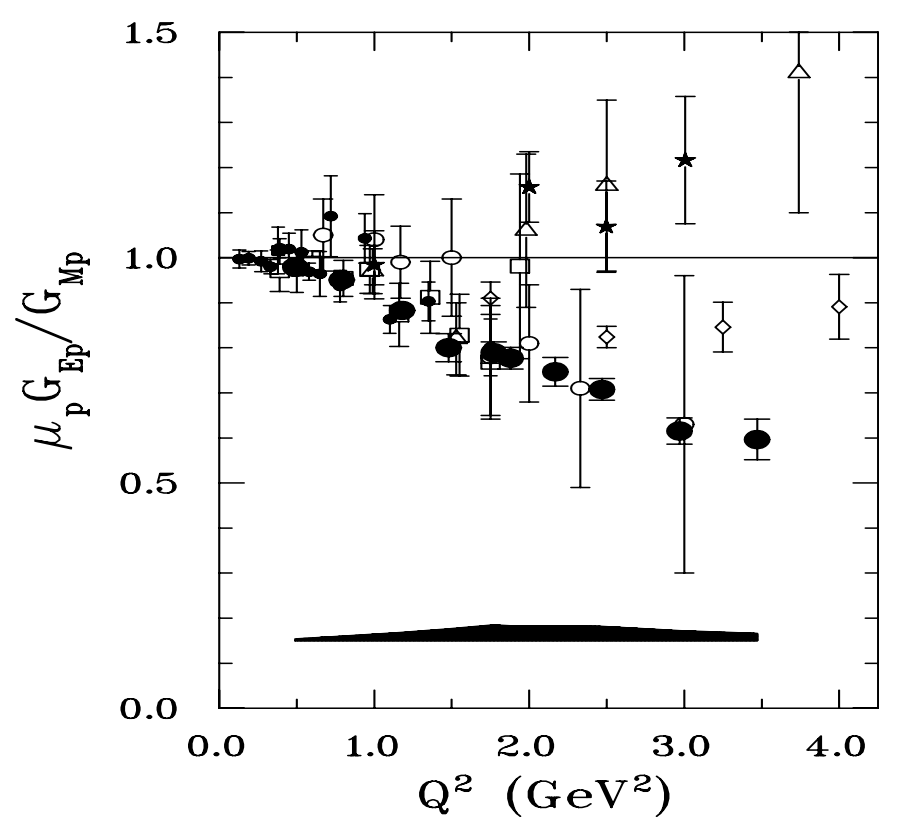

Fig. 1. The Jefferson Lab data [18] on the ratio $G_{E}^{p} / G_{M}^{p}$ showing the discrepancy between the recoil polarization (solid circles) and the Rosenbluth (other symbols) techniques.

\section{Evidence for multiple photon effects in elastic electron scattering from the proton}

Essentially all electron scattering experiments to study proton and nuclear structure to date have been analyzed in terms of single photon exchange. The fine structure coupling constant $\alpha \sim 1 / 137$ is small enough that leading order has been adequate. There are a few specific examples where multiple photon exchange is known to be significant, e.g. in comparison of electron and positron scattering in kinematics where the single photon exchange cross-section is small, or in radiative processes. Thus, it came as a surprise when the Jefferson Lab Hall A recoil polarization measurements of electron-proton elastic scattering at momentum transfers of about $2(\mathrm{GeV} / c)^{2}[18]$ showed a substantial deviation from the data obtained over several decades with the Rosenbluth technique [19], which is based on precise cross-section measurements. This discrepancy has been interpreted as the effect of multiple photon exchange in the elastic electron-proton crosssection [20]. The cross section for elastic electron-proton scattering in the one-photon exchange approximation can be written in terms of the pointlike Mott cross-section, the Sachs form factors $G_{E}^{p}$ and $G_{M}^{p}$ and the electron scattering angle $\theta$ as

$$
\frac{\mathrm{d} \sigma}{\mathrm{d} \Omega}=\left(\frac{\mathrm{d} \sigma}{\mathrm{d} \Omega}\right)_{M o t t} \cdot\left[\frac{G_{E}^{p 2}+\tau G_{M}^{p 2}}{1+\tau}+2 \tau G_{M}^{p 2} \tan ^{2} \frac{\theta}{2}\right]
$$

where $\tau=Q^{2} / 4 M^{2}$. Figure 1 shows the recoil polarization determination of $G_{E}^{p} / G_{M}^{p}$ (solid circles) as a function of momentum transfer $Q^{2}$. The Rosenbluth data (all other data points) are believed to be uncorrected for the effects

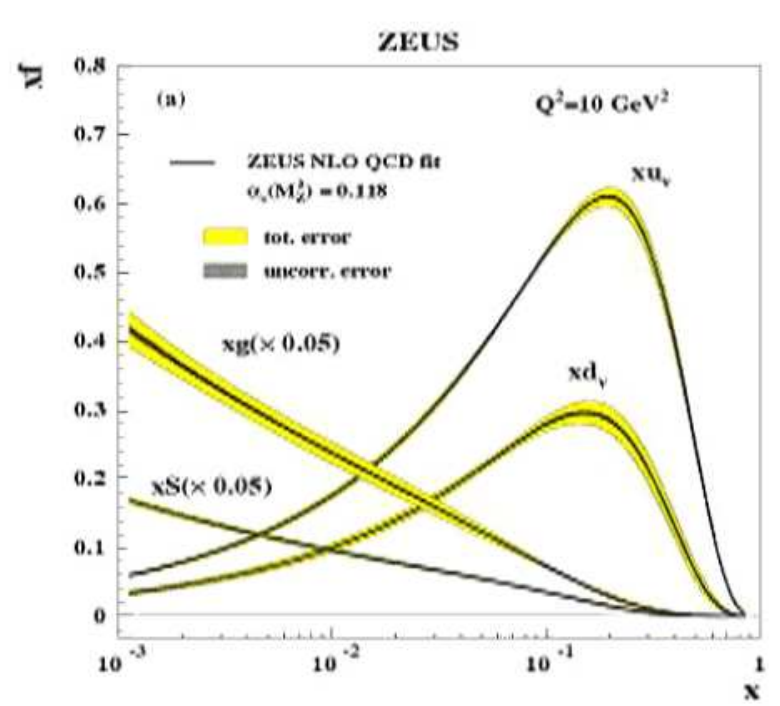

Fig. 2. The quark and gluon momentum distributions at $Q^{2}=10(\mathrm{GeV} / c)^{2}$ as a function of parton momentum $x$ as determined by the ZEUS experiment [23] at the HERA electronproton collider. Note that the sea quark momentum $x S$ and the gluon momentum $x g$ distributions are divided by a factor of 20 .

of multiple photon exchange and so give an incorrect determination at higher $Q^{2}$, i.e. above about $1(\mathrm{GeV} / c)^{2}$.

This multiple photon exchange contribution to elastic electron-proton scattering can be confirmed by precise comparison of electron-proton with positron proton elastic scattering or by measurement of the asymmetry $A_{y}$ in scattering of unpolarized electrons from a vertically polarized proton target [21]. If confirmed, this is a very significant result.

\section{Role of sea quarks in nucleon structure}

QCD tells us that the nucleon comprises three valence quarks and a sea of quark-antiquark pairs. From the earliest days of nuclear physics, these sea quarks in the form of mesons, have been viewed as playing an important role in the long distance structure of the nucleon e.g. the magnitude and sign of the proton and neutron magnetic moments. In addition, the most successful hadronic theoretical descriptions of light nuclei incorporate meson exchange between nucleons as an essential element of nuclear binding. This "meson cloud" structure to the nucleon has generally been accepted but has lacked both a rigorous theoretical underpinning and a definitive quantitative basis from experiment.

The role of valence quarks in nucleon structure has been studied extensively. The effects of sea quarks and gluons are relatively poorly determined, in large part because they require high center-of-mass energy, and are a major focus of interest for the future [22]. One of the important contributions over the last decade has been the experimental measurement of deep inelastic scattering at high energies to determine the effects of the sea quarks and 


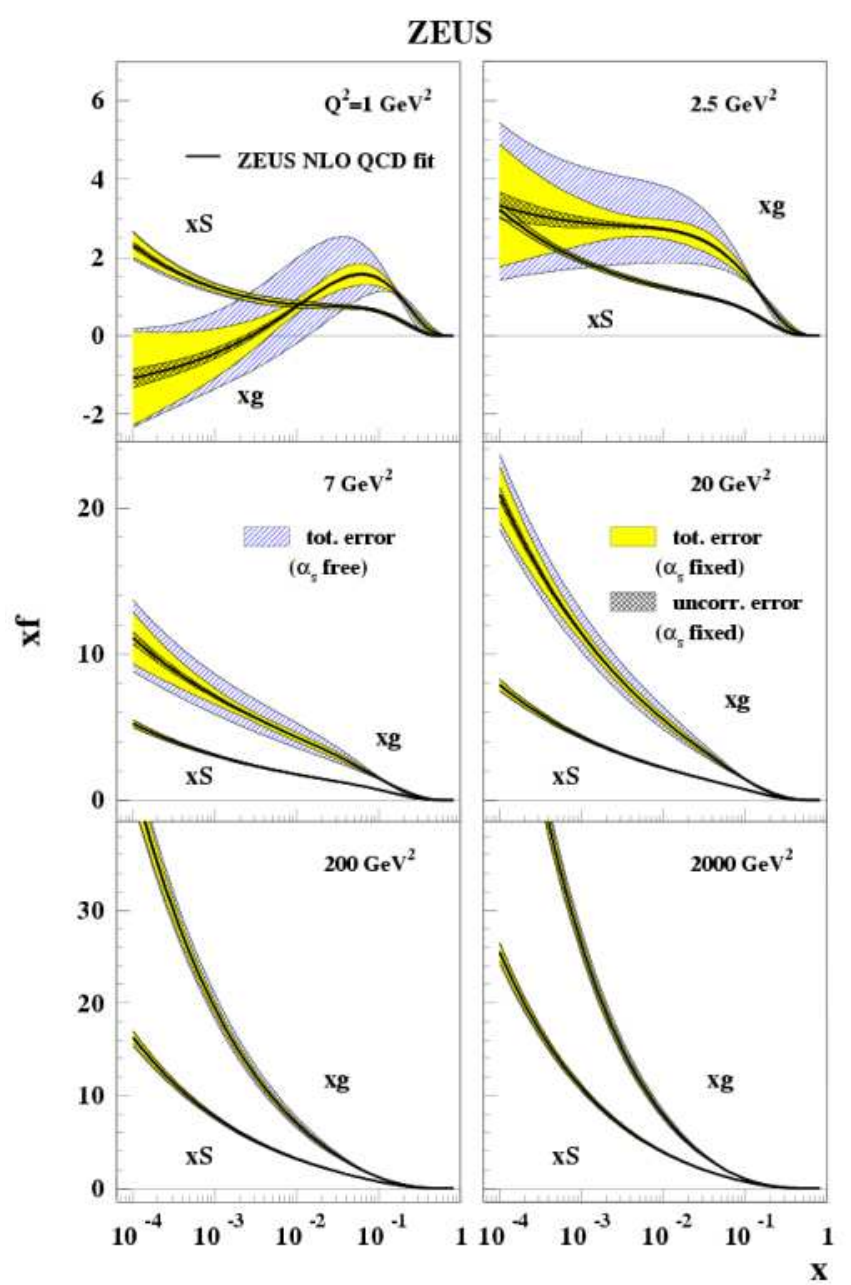

Fig. 3. Comparison of the gluon and sea distributions from the ZEUS-S NLO QCD fit for various $Q^{2}$ values [23] as measured at the HERA electron-proton collider.

gluons. In particular, data taken by experiments at the HERA electron-proton collider [23] have for the first time allowed a determination of the gluon momentum distribution in the proton, as shown in fig. 2. The QCD evolution of HERA data [23] shows a significant sea contribution at low $Q^{2}$, in contrast to the gluon contribution which vanishes, as seen in fig. 3. This supports the point of view of a strong role for sea quarks at low $Q^{2}$.

At low energies, electron scattering experiments determine the elastic electric and magnetic form factors of the proton and neutron. Friedrich and Walcher have postulated that the $Q^{2}$ dependence of the elastic form factors in the region 0.1 to $0.5(\mathrm{GeV} / c)^{2}$ may be sensitive to the meson cloud structure of the nucleon and have produced parameterizations of world data which suggest that there may be experimental support for this ansatz [24]. They fit the measured four form factors with a parameterization which consists of a smooth contribution and a bump contribution. Figure 4 shows the world's data for the proton elastic form factor plotted as a function of momentum transfer $Q^{2}$, where the smooth contribution is subtracted.

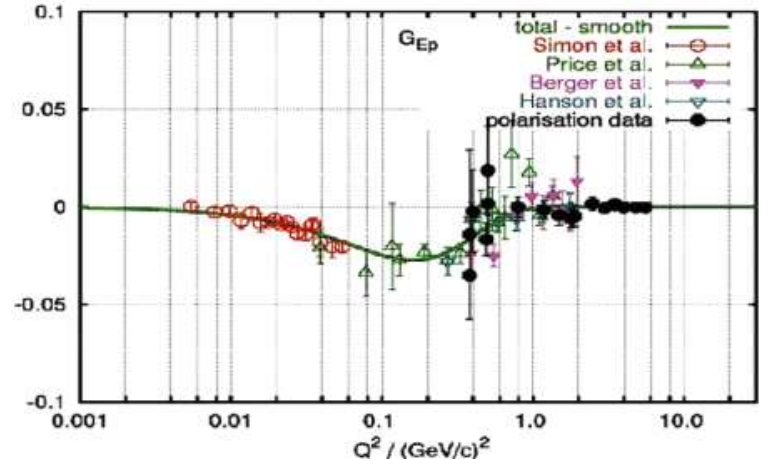

Fig. 4. The proton charge elastic form-factor with the smooth contribution subtracted in the parameterization of Friedrich and Walcher [24].

A $2 \%$ dip in the parameterization is obvious at $Q^{2} \sim 0.1-$ $0.2(\mathrm{GeV} / c)^{2}$, which coincides with the location of the peak in the neutron charge elastic form-factor $\mathrm{G}_{E}^{n}$. In the absence of realistic QCD calculations, it is hard to definitively state that this structure at low $Q^{2}$ is due to the meson cloud structure of the nucleon. However, it is a physically plausible explanation.

\section{BLAST Experiment at MIT-Bates}

A new set of precision measurements of the low $Q^{2}$ elastic form factors of the proton and neutron have been carried out using the South Hall Ring (SHR) at the MIT-Bates Linear Accelerator Center. The Bates Large Acceptance Spectrometer Toroid (BLAST) was constructed [25] to detect scattered electrons, protons, neutrons and pions in the scattering of longitudinally polarized electrons with an energy of $850 \mathrm{MeV}$ from polarized targets of hydrogen and deuterium. The polarized internal gas target technique offers minimal systematic uncertainties and a high statistics sample of data were taken by the BLAST experiment over an eighteen month period from late 2003 to mid 2005.

The BLAST data are under analysis and will be able to provide new and independent experimental constraints of the Friedrich-Walcher ansatz.

The polarized protons and deuterons (both vector and tensor) were produced using an Atomic Beam Source (ABS) [26], which was located in the substantial and spatially varying magnetic field of the BLAST toroid. The target spin state was alternated every five minutes by switching the final RF transition immediately before the target to ensure equal target densities for each of the three states (vector + , vector - , tensor - ). The electrons scattered from the polarized protons and deuterons in a cylindrical, windowless aluminum target tube $600 \mathrm{~mm}$ long, $15 \mathrm{~mm}$ in diameter and with a wall thickness of $50 \mu \mathrm{m}$. The polarized target was tuned and monitored using a Breit-Rabi system which continuously sampled the atomic polarization of a small fraction of the incoming beam from the ABS. The vector polarizations of both the proton and deuteron was typically 0.75 . Data were taken with stored electron beam intensities up to $225 \mathrm{~mA}$. 


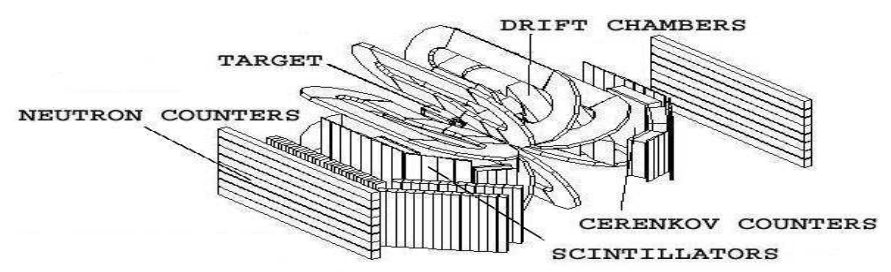

Fig. 5. A schematic layout of the BLAST experiment at MITBates.
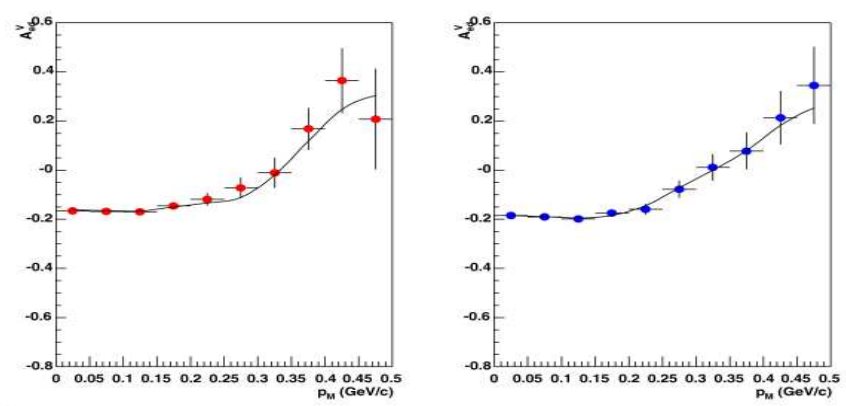

Fig. 6. The vector asymmetry $A_{e d}^{V}$ in quasielastic (e, e'p) scattering from vector polarized deuterium as a function of missing momentum $p_{m}$ for $0.1<Q^{2}<0.2(\mathrm{GeV} / c)^{2}$, as measured by the BLAST experiment [27].

The polarized electron beam originated from a GaAs polarized electron source and the storage ring was filled with alternating electron polarizations approximately every half hour. The longitudinal beam polarization at the target was maintained using a Siberian Snake solenoid system. The beam polarization was continuously monitored using a laser Compton backscattering polarimeter, located upstream of the injection point in the SHR. The average beam polarization over the BLAST data taking period was 0.65 .

The BLAST (see fig. 5) consisted of eight copper coils which provided a 0.4 Tesla toroidal magnetic field. For these measurements it was instrumented with symmetric detectors in the horizontal plane: three drift chambers for momentum, angle and position determination; plastic scintillators for triggering and time of flight, and Cerenkov detectors for pion rejection. In addition, large plastic scintillators for neutron detection were arranged on one side. The background rate for scattering from the target cell was measured and found to be negligible.

Figure 6 shows a fraction of the BLAST data acquired in quasielastic (e, $\left.\mathrm{e}^{\prime} \mathrm{p}\right)$ scattering from vector polarized deuterium [27]. The scattering asymmetry $A_{e d}^{V}$ is plotted as a function of the missing momentum $\left(p_{m}\right)$ of the proton in the nucleon. The solid curve is the predic- tion of a Monte Carlo simulation which uses Arenhövel's theory [28] as well as a realistic description of the experiment. At low $p_{m}$, the scattering is dominated by the $S$ state in deuterium and the asymmetry is very close to that for scattering from a free proton. These data can be used to determine the product of beam and target vector polarization. At high $p_{m}$, the scattering is dominated by the $D$-state in the deuteron, where both proton and neutron spins are anti-aligned with respect to the nuclear spin. Thus, the scattering asymmetry changes sign. The $p_{m}$ range of the data extend out to $500 \mathrm{MeV} / c$. BLAST data on the four elastic form factors of the proton and neutron are expected to be published in 2006. In addition, a sizable data set on electron scattering from tensor polarized deuterium was acquired with BLAST.

\section{Conclusion}

The electromagnetic probe provides a beautiful and precise means to study strongly interacting matter. We are fortunate to witness great advances in accelerator and experimental technology so that the full power of the electromagnetic probe can be exploited to study hadronic matter. The two examples discussed above indicate that new insight into Nature is being provided by the elementary elastic electron nucleon scattering reaction, particularly with spin polarization techniques.

The role of the sea quarks/meson cloud in nucleon structure continues to be a subject of significant interest. Precision determination of the elastic form factors at low momentum transfers from BLAST may confirm the ansatz of Friedrich and Walcher. Confirmation of a dip in the proton electric and magnetic form factors as well as the neutron magnetic form factor at $Q^{2} \sim 0.15(\mathrm{GeV} / c)^{2}$ will not definitively quantify the role of the meson cloud but it will demand of theorists a convincing explanation. I note that the recent G0 data [29] on the linear combination of the electric and magnetic strange form factors of the proton suggest a $Q^{2}$ dependence at similar values of $Q^{2}$ to that of the dip. Is this significant? Clearly, more precise data are needed.

The determination of $G_{E}^{n}$ as a function of $Q^{2}$ by many laboratories over a decade has clearly been a triumph for the field of electromagnetic nuclear physics. With the BLAST data, it is expected that this quantity will be determined to better than $\pm 5 \%$ at low momentum transfers. It is anticipated that this will quantitatively constrain the meson cloud contribution to the charge distribution of the neutron.

The experimental and theoretical contributions at MAMI, particularly by our five distinguished colleagues who are honored here, have been important to the significant progress made worldwide. It has been a pleasure and a privilege to be part of this unique celebration. I congratulate Profs. H. Arenhövel, H. Backe, D. Drechsel, J. Freidrich, K.-H. Kasier, and Th. Walcher on their distinguished careers and I wish them every success in the next phase of their lives. 
The author would like to acknowledge discussions with A.M. Bernstein, T.W. Donnelly, R. Miskamen, A.H. Mueller, J.W. Negele, and C.N. Papanicolas. In addition, the author would like to acknowledge that the BLAST experiment is the fruit of a dedicated collaboration over an extended period of time. In particular, an outstanding cohort of graduate students is playing an essential role. The author's research is supported by the United States Department of Energy under the Cooperative Agreement DE-FG02-94ER40818.

\section{References}

1. R.G. Edwards et al., Phys. Rev. Lett. 96, 052001 (2006).

2. D. Rohe et al., Phys. Rev. Lett. 83, 4257 (1999).

3. T. Eden et al., Phys. Rev. C 50, R1749 (1994).

4. I. Passchier et al., Phys. Rev. Lett. 82, 4988 (1999).

5. R. Madey et al., Phys. Rev. Lett. 92, 122002 (2003).

6. N. Sparveris et al., Phys. Rev. Lett. 94, 122003 (2005).

7. V. Pascalutsa, M. Vanderhaeghen, Phys. Rev. Lett. 95, 232001 (2005).

8. C. Alexandrou et al., Phys. Rev. Lett. 94, 021601 (2005).

9. J. Roche et al., Phys. Rev. Lett. 85, 708 (2000).

10. G. Laveissiere et al., Phys. Rev. Lett. 93, 122001 (2004).

11. P. Bourgeois et al., submitted to Phys. Rev. Lett. (April 2006).

12. A. Airapetian et al., Phys. Rev. D 71, 012003 (2005).

13. X. Zheng et al., Phys. Rev. C 70, 065207 (2004).
14. E.S. Ageev et al., Phys. Lett. B 633, 25 (2006).

15. J. Kiryluk (MIT) for the STAR collaboration, Proceedings of PANIC 2005, October 2005, Santa Fe, New Mex$i c o$; K. Boyle (Stony Brook) for the PHENIX collaboration, Proceedings of PANIC 2005, October 2005, Santa Fe, New Mexico, to be published by the American Institute of Physics.

16. S. Kowalski, these proceedings.

17. F. Maas, these proceedings.

18. V. Punjabi et al., Phys. Rev. C 71, 055202 (2005).

19. I.A. Qattan et al., Phys. Rev. Lett. 94 (142301) (2005).

20. P.A.M. Guichon, M. Vanderhaeghen, Phys. Rev. Lett. 91, 142303 (2003); P.G. Blunden, W. Melnitchouk, J.A. Tjon, Phys. Rev. Lett. 91, 142304 (2003).

21. A.A. Afanasev et al., Phys. Rev. D 72, 013008 (2005).

22. A. Deshpande, R. Milner, R. Venugopalan, W. Vogelsang, Annu. Rev. Nucl. Part. Sci. 55, 165 (2005).

23. S. Chekanov et al., Phys. Rev. D 67, 012007 (2002).

24. J. Friedrich, Th. Walcher, Eur. Phys. J. A 17, 607 (2003).

25. BLAST Technical Design Report August 10th, 1997.

26. D. Cheever et al., Nucl. Instrum. Methods A 556, 410 (2006).

27. A. Maschinot, MIT PhD Thesis 2005 (unpublished).

28. H. Arenhövel, W. Leidemann, E.L. Tomusiak, Phys. Rev. C 52, 1232 (1995); 46, 455 (1992); Z. Phys. A 331, 123 (1988); 334, 363 (1989).

29. The G0 Collaboration, Phys. Rev. Lett. 95, 092001 (2005). 